correct, but following the successful Diels-Alder reaction, a marvellous series of transformations ensued that resulted in the construction of the entire octacyclic scaffold (Fig. 1b).

It is suggested that the high regiocontrol and diastereoselectivity of the Diels-Alder reaction can be explained by molecular orbital interactions and a crucial hydrogen bond between the free hydroxyl group of the diene and the more Lewis basic carbonyl of the quinone dienophile (Fig. 1b), which correctly oriented the reactants. The product undergoes subsequent hemiketal formation to generate ring $\mathrm{D}$, thus leaving the resulting hydroxyl group in a favourable conformation to enable a cyclization reaction involving an intramolecular nucleophilic ipso substitution. Overall, this remarkable cascade reaction installed three rings and 4 of 5 stereogenic centres, all in $98 \%$ yield.

The elegant synthetic approach ultimately facilitated the construction of both enantiomers and determination of the absolute stereochemistry as (+)-BE43472B. The impressive synthesis now sets the stage to answer other important questions. For example, what is the mechanism of action of these unusual antibiotics? Are they active in vivo? What structural modifications will provide improved pharmacological properties? Answers are now closer than ever.

The work is an example of the increasing power of cascade reactions in chemical synthesis. Clearly there are significant advantages to executing a series of 'one pot' transformations with stereochemical precision. In the present case, this welldesigned approach will now enable the swift creation of congeners to probe structureactivity relationships.

Harvesting antibiotics such as (+)-BE43472B from nature is part of the equation for satisfying the need for new therapeutics. But fermentation techniques and semi-synthesis are limited in the breadth of related structures that can be produced. In order to fully explore and comprehend the newly found chemical space of such compounds, total synthesis is a critical component. The inspired work by the Nicolaou group now invites the creation of analogues so that this new chemical class of antibiotics can be investigated.

David C. Rowley is in the Department of Biomedical and Pharmaceutical Sciences, College of Pharmacy, University of Rhode Island, Kingston, Rhode Island 02881, USA.

e-mail:drowley@uri.edu

References

1. Nicolaou, K. C., Lin, Y. H. \& Becker, J. Angew. Chem. Int. Ed doi:10.1002/anie.200900058 (2009).

2. Bentley, S. D. et al. Nature 417, 141-147 (2002)

3. Ikeda, H. et al. Nature Biotechnol. 21, 526-531 (2003).

4. Fenical, W. \& Jensen, P. R. Nature Chem. Biol. 2, 666-673 (2006)

5. Corey, E. J. et al. J. Am. Chem. Soc. 100, 4620-4622 (1978).

6. Newman, D. J. \& Cragg, G. M. J. Nat. Prod. 70, 461-477 (2007).

7. Kushida, H. et al. Antitumoric BE43472 manufacture with Strptomyces. JPO patent 8,143,569 (1996).

8. Socha, A. M. et al. J. Nat. Prod. 69, 1070-1073 (2006).

9. Socha, A. M. et al. Bioorg. Med. Chem. 14, 8446-8454 (2006).

\title{
ERRATUM
}

\section{Powering the planet with solar fuel}

\author{
Harry B. Gray
}

Nature Chemistry 1, 7 (2009); corrected after print: 19 March 2009.

In the version of this article originally published, the ratio of hydrogen to oxygen given in the figure caption was incorrect; it should have read 2:1, and has now been corrected in the HTML and PDF versions. 